\title{
THEORETICAL AND PRACTICAL MECHANICS
}

УДК 534/539:[57+61]

\section{ОЦЕНКА ТОАЩИНЫ ХРЯЩЕВОГО ТРАНСПААНТАТА АЛЯ ТИМПАНАЛЬНОЙ МЕМБРАНЫ ПРИ УАААЕНИИ РЕТРАКЦИОННОГО КАРМАНА (КОНЕЧНО-ЭАЕМЕНТНОЕ МОАЕАИРОВАНИЕ)}

\author{
С. М. БОСЯКОВ ${ }^{1)}$ К. С. ЮРКЕВИЧ ${ }^{1)}$, \\ Г. И. МИХАСЕВ ${ }^{1)}$, Л. Г. ПЕТРОВА ${ }^{2)}$, М. М. МАЙСЮК ${ }^{3)}$
}

${ }^{1)}$ Белорусский государственный университет, пр. Независимости, 4, 220030, г. Минск, Беларусь

${ }^{2)}$ Белорусская медицинская академия последипломного образования, ул. П. Бровки, 3, корп. 3, 220013, г. Минск, Беларусь

${ }^{3)}$ Республиканский научно-практический ичентр оториноларингологии, ул. Сухая, 8, 220004, г. Минск, Беларусь

Возникновение ретракционного кармана тимпанальной мембраны (барабанной перепонки) влечет за собой нарушение звуковой проводимости среднего уха. Хирургическое удаление фиксированных ретракционных карманов

\section{Образец цитирования:}

Босяков СМ, Юркевич КС, Михасев ГИ, Петрова ЛГ, Майсюк ММ. Оценка толщины хрящевого трансплантата для тимпанальной мембраны при удалении ретракционного кармана (конечно-элементное моделирование). Журнал Белорусского государственного университета. Математика. Информатика. 2021;1:69-78.

https://doi.org/10.33581/2520-6508-2021-1-69-78

\section{For citation:}

Bosiakov SM, Yurkevich KS, Mikhasev GI, Petrova LG, Maisyuk MM. Assessment of the cartilage transplant thickness after removing of the tympanic membrane retraction pocket (finiteelement modelling). Journal of the Belarusian State University. Mathematics and Informatics. 2021;1:69-78. Russian. https://doi.org/10.33581/2520-6508-2021-1-69-78

\section{Авторы:}

Сергей Михайлович Босяков - доктор физико-математических наук, доцент; декан механико-математического факультета.

Кирилл Сергеевич Юркевич - кандидат физико-математических наук, доцент; доцент кафедры био- и наномеханики механико-математического факультета.

Геннадий Иванович Михасев - доктор физико-математических наук, профессор; заведующий кафедрой био- и наномеханики механико-математического факультета.

Людмила Григорьевна Петрова - доктор медицинских наук, профессор; заведующий кафедрой оториноларингологии хирургического факультета.

Марина Михайловна Майсюк - кандидат медицинских наук; заведующий оториноларингологическим отделением для детей стационара.

\footnotetext{
Authors:

Sergei M. Bosiakov, doctor of science (physics and mathematics), docent; dean of the faculty of mechanics and mathematics. bosiakov@bsu.by

https://orcid.org/0000-0003-4524-9304

Kirill S. Yurkevich, $\mathrm{PhD}$ (physics and mathematics), docent; associate professor at the department of bio- and nanomechanics, faculty of mechanics and mathematics.

yurkevichks@bsu.by

Gennady I. Mikhasev, doctor of science (physics and mathematics), full professor; head of the department of bio- and nanomechanics, faculty of mechanics and mathematics. mikhasev@bsu.by

https://orcid.org/0000-0002-9409-9210

Lyudmila G. Petrova, doctor of science (medicine), full professor; head of the department of otorhinolaryngology, faculty of surgery. bsm21@mail.ru

Marina M. Maisyuk, $\mathrm{PhD}$ (medicine); head of the department of otorhinolaryngology for children in-patient hospital. merylmmm@mail.ru
} 
приводит к появлению перфораций, на место которых устанавливается хрящевой трансплантат. Целью работы являлось определение геометрических параметров хрящевого трансплантата, обеспечивающих звуковую проводимость колебательной системы среднего уха, соответствующую слуховым функциям среднего уха в норме. Определение геометрических параметров трансплантата осуществлялось на основании соответствующей конечно-элементной модели. В качестве величин, характеризующих слуховую проводимость колебательной системы среднего уха, рассматривались собственные частоты свободных колебаний. На основании сравнительного анализа спектров собственных частот свободных колебаний среднего уха в норме и среднего уха с хрящевыми трансплантатами различной толщины установлено, что толщина трансплантата, накладываемого на задневерхний квадрант после удаления фиксированного ретракционного кармана, составляет $0,193 \pm 0,031$ мм. Полученные результаты могут быть использованы при планировании хирургических операций по восстановлению целостности барабанной перепонки и улучшению слуховой проводимости.

Ключевые слова: среднее ухо; тимпанальная мембрана; конечно-элементное моделирование; хрящевой трансплантат; частота свободных колебаний; звуковая проводимость.

Благодарность. Работа выполнена при поддержке гранта Президента Республики Беларусь в области науки.

\title{
ASSESSMENT OF THE CARTILAGE TRANSPLANT THICKNESS AFTER REMOVING OF THE TYMPANIC MEMBRANE RETRACTION POCKET (FINITE-ELEMENT MODELLING)
}

\author{
S. M. BOSIAKOV ${ }^{\mathrm{a}}$, K. S. YURKEVICH ${ }^{\mathrm{a}}$, \\ G. I. MIKHASEV ${ }^{\mathrm{a}}$, L. G. PETROVA ${ }^{\mathrm{b}}$, M. M. MAISYUK ${ }^{\mathrm{c}}$ \\ ${ }^{a}$ Belarusian State University, 4 Niezaliežnasci Avenue, Minsk 220030, Belarus \\ ${ }^{\mathrm{b}}$ Belarusian Medical Academy of Post-Graduate Education, \\ 3 P. Broǔki Street, 3 building, Minsk 220013, Belarus \\ ${ }^{\mathrm{c}}$ Republican Scientific and Practical Centre of Otolaryngology, \\ 8 Suhaja Street, Minsk 220004, Belarus \\ Corresponding author: S. M. Bosiakov(bosiakov@bsu.by)
}

The retraction pocket emergence of the tympanic membrane (eardrum) leads to the sound conduction disorder of the middle ear. Surgical removal of fixed retraction pockets leads to perforations. A cartilage grafts in the region of these perforations are installed. The aim of the study is to assess the geometric parameters of the cartilage graft, providing sound conductivity of the middle ear oscillatory system corresponding the auditory functions of the normal middle ear. The evaluation of the geometric parameters of the cartilage graft is carried out on the basis of the middle ear finite-element model. The eigenfrequencies are utilised as quantities characterising the auditory conductivity of the middle ear oscillatory system. The thickness of the graft attached on the posterosuperior quadrant after removal of the fixed retraction pocket is $0.193 \pm 0.031 \mathrm{~mm}$. It is evaluated on basis of comparative analysis of the middle ear eigenfrequency spectra in normal conditions, the middle ear with attached cartilaginous grafts of different thicknesses. The obtained results can be used for planning of surgical operations to restore the integrity of the tympanic membrane and improve auditory conductivity.

Keywords: middle ear; tympanic membrane; finite-element modelling; cartilage graft; eigenfrequency; auditory conductivity.

Acknowledgements. This study is supported by a grant from the President of the Republic of Belarus in the field of science.

\section{Введение}

Одним из нарушений звуковой проводимости среднего уха в сочетании с хроническим средним отитом является возникновение ретракционного кармана области pars tensa тимпанальной мембраны (патологическое выдувание барабанной перепонки в тимпанальной полости) [1]. Самый распространенный участок образования ретракционного кармана - задневерхний квадрант тимпанальной мембраны, поскольку средний фиброзный слой задневерхнего квадранта области pars tensa имеет наиболее ослабленную структуру по сравнению с более плотными передним и задненижним квадрантами [2]. Развитие такой патологии может привести к повреждению костной ткани молоточка и стремени, а также к возникновению холестеатомы [3]. Ранние или небольшие ретракционные карманы могут быть полностью устранены медикаментозным лечением, тогда как фиксированные ретракционные карманы обычно 
требуют хирургического вмешательства, чтобы предотвратить дальнейшее развитие патологии и скорректировать анатомические или функциональные изменения. После удаления ретракционного кармана возникают перфорации тимпанальной мембраны, которые очень редко подвергаются самопроизвольному заживлению. В большинстве случаев на область перфорации устанавливается хрящевой трансплантат из козелка или ушной раковины [4; 5]. Использование такого трансплантата в реконструкции барабанной перепонки имеет ряд преимуществ перед применением мембранных трансплантатов, но может изменять акустические характеристики колебательной системы среднего уха.

Влияние геометрических параметров хрящевого трансплантата на звуковую проводимость колебательной системы среднего уха анализировалось в экспериментальных и теоретических работах [6-14].

В исследовании [6] определены амплитуды колебаний хрящевых трансплантатов различного типа (хрящевых пластин, хрящевых палисадов и островковых трансплантатов), возникающих при действии звукового давления. Для этого использована сканирующая лазерная доплеровская установка, позволяющая определить амплитуды вынужденных колебаний трансплантата в модели ущной канал - барабанная перепонка. На основании частотных характеристик и величин перемещений точек барабанной перепонки и трансплантатов сделан вывод, что предпочтительной с позиций акустики является установка сплошной хрящевой пластины толщиной менее 0,5 мм по сравнению с применением островковых трансплантатов и хрящевых палисадов.

В работе [7] на базе электроакустической модели проведен анализ влияния локализации протеза, устанавливаемого на реконструированной тимпанальной мембране, на усилие в сочленении протез стремя, а также на смещение и поворот основания стременной косточки. Математические модели для расчета состояния барабанной перепонки, определения напряжений и собственных частот колебаний барабанной перепонки в норме и при патологии при различных значениях отрицательного звукового давления представлены в работе [8]. Здесь также приведены результаты анализа влияния на механические характеристики барабанной перепонки изменения жесткости обеих внутрибарабанных мышц и фенестрации перепонки.

Разработке метода биомеханического моделирования структур среднего уха в пакетах прикладных программ в норме, при патологических изменениях, коррекции и реконструкции посвящено исследование [9]. Апробация метода выполнена на конкретных примерах с использованием конечно-элементных пакетов прикладных программ SolidWorks и COSMOSWorks. В работах [12-14] с применением конечно-элементного моделирования определены акустические характеристики хрящевого трансплантата (хрящевой пластины), используемого при различных формах и расположениях областей перфорации тимпанальной мембраны. Установлено, что хрящевая пластина размером от 0,1 до 0,2 мм представляется оптимальной с точки зрения вибрации барабанной перепонки. Толщина трансплантата, равная 0,2 и 0,1 мм, является предпочтительной исходя из критерия механической жесткости и потерь при передаче звука на более низких и высоких частотах соответственно. Настоящая работа развивает это направление исследований, она посвящена определению геометрических параметров хрящевого трансплантата, устанавливаемого на область задневерхнего квадранта барабанной перепонки после удаления ретракционного кармана.

\section{Материалы и методы исследования}

Разработка твердотельных моделей тимпанальной мембраны, молоточка, наковальни и стремени на основании томографических данных описана в работах $[15 ; 16]$ с учетом того, что тимпанальная мембрана имеет слоистую структуру [17]. Наиболее тонкая часть, соответствующая области pars flaccida, имеет толщину 30 мкм, толщина задневерхнего квадранта области pars tensa составляет 60 мкм, остальная часть области pars tensa равна 90 мкм. Тимпанальная мембрана закреплена в тимпанальном кольце, которое жестко заделано по контуру. Закрепление отсутствует в передней части тимпанальной мембраны, где тимпанальное кольцо разделяет ее на две области - pars tensa и pars flaccida [18]. Кроме того, в модели к костям среднего уха в соответствующих точках прикреплены тензорное сухожилие тимпанальной мембраны и стремени, латеральная, передняя и верхняя молоточковые связки, задняя и передняя наковальневые связки, кольцевая стапедиальная связка, а также тензорная тимпанальная и стапедиальная мышцы. Согласно [19] связки и сухожилия моделируются с использованием цилиндрических форм. Длина и радиус каждой из связок среднего уха составляют 0,8 и 0,5 мм соответственно. Длина тензорной мышцы барабанной перепонки равна 3,0 мм, радиус ее поперечного сечения $-0,5$ мм, длина и радиус поперечного сечения стапедиальной мышцы - 2,0 и 0,43 мм соответственно [12; 13]. Моделирование жесткости кольцевой стапедиальной связки в плоскости основания (подножной пластинки) стремени выполнено с использованием равномерно распределенных по внешнему контуру 25 линейных пружинных элементов. Жесткость каждой пружины составляет 40 H/м [20]. Твердотельная модель среднего уха представлена на рис. 1 . 
$a / a$

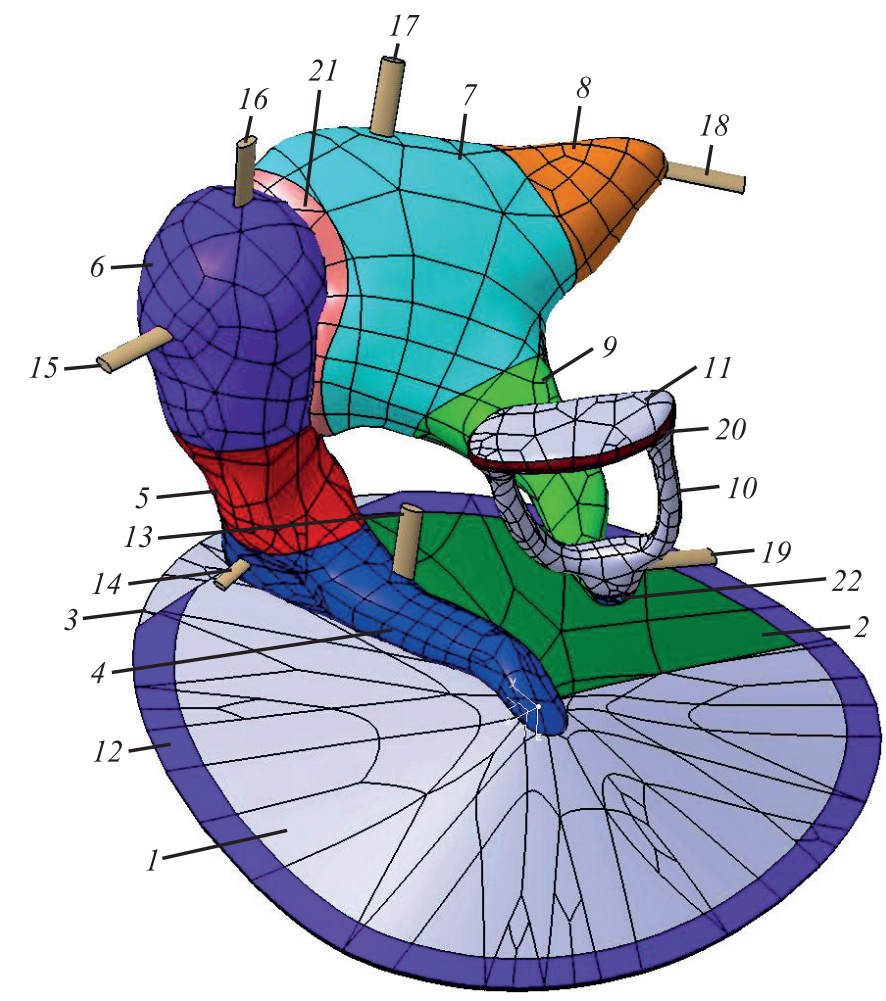

$\sigma / b$

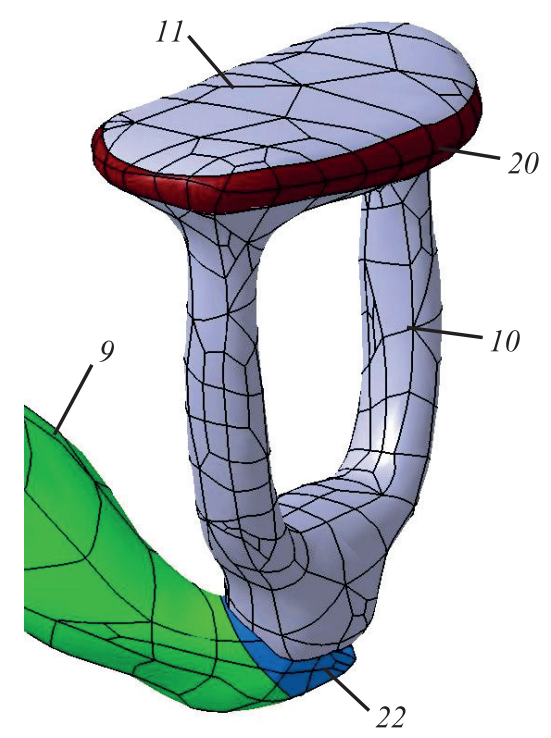

Puc. 1. Твердотельная модель среднего уха (a) и область наковально-стременного сустава (б):

1 - область pars tensa тимпанальной мембраны; 2 - задневерхний квадрант тимпанальной мембраны; 3 - область pars flaccida тимпанальной мембраны; 4 - рукоятка молоточка; 5 - шейка молоточка; 6 - головка молоточка; 7 - тело наковальни;

8 - короткий отросток наковальни; 9 - длинный отросток наковальни; 10 - стремя;

11 - основание (подножная пластинка) стремени; 12 - тимпанальное кольцо;

13 - tensor tympani (сухожилие, натягивающее барабанную перепонку); 14 - передняя молоточковая связка;

15 - задняя (латеральная) молоточковая связка; 16 - верхняя молоточковая связка;

17 - передняя связка наковальни; 18 - задняя связка наковальни; 19 - сухожилие стремени;

20 - кольцевая связка стремени; 21 - наковально-молоточковый сустав; 22 - наковально-стременной сустав

Fig. 1. Biomechanical model of the middle ear $(a)$ and the incudostapedial joint region $(b)$ :

1 - pars tensa; 2 - posterosuperior quadrant of the tympanic membrane; 3 - pars flaccida; 4 - manubrium; 5 - malleus neck;

6 - malleus head; 7 - incus body; 8 - short process of the incus; 9 - long process of the incus; 10 - stapes;

11 - stapes footplate; 12 - tympanic annulus; 13 - tensor tympani; 14 - anterior mallear ligament;

15 - posterior (lateral) mallear ligament; 16 - superior mallear ligament; 17 - anterior incudal ligament; 18 - posterior incudal ligament; 19 - stapedial tendon; 20 - stapedial annular ligament;

$$
21 \text { - incudomalleolar joint; } 22 \text { - incudostapedial joint }
$$

Геометрические параметры элементов твердотельной модели среднего уха приведены в табл. 1. Они с высокой точностью соответствуют данным, принимаемым в работах [20-23] для аналогичных моделей.

Таблица 1

Характерные геометрические размеры твердотельной модели среднего уха

Table 1

Typical geometric parameters

of the biomechanical model of the middle ear

\begin{tabular}{|l|c|}
\hline \multicolumn{1}{|c|}{ Параметр } & Значение \\
\hline Диаметр тимпанальной мембраны вдоль рукоятки молоточка, мм & 10,0 \\
\hline $\begin{array}{l}\text { Диаметр тимпанальной мембраны, перпендикулярный } \\
\text { рукоятке молоточка, мм }\end{array}$ & 9,0 \\
\hline Высота конуса тимпанальной мембраны, мм & 1,5 \\
\hline Площадь поверхности тимпанальной мембраны, мм² & 74,09 \\
\hline
\end{tabular}


Теоретическая и прикладная механика Theoretical and Practical Mechanics

Окончание табл. 1

Ending table 1

\begin{tabular}{|l|c|}
\hline \multicolumn{1}{|c|}{ Параметр } & Значение \\
\hline Диапазон толщин области pars tensa, мм & $0,06-0,09$ \\
\hline Длина рукоятки молоточка до конца бокового отростка, мм & 4,36 \\
\hline Длина от бокового отростка до конца головки молоточка, мм & 4,50 \\
\hline Длина длинного отростка наковальни, мм & 7,54 \\
\hline Длина короткого отростка наковальни, мм & 4,87 \\
\hline Высота стремени, мм & 2,69 \\
\hline Длина основания стремени, мм & 2,64 \\
\hline Ширина основания стремени, мм & 1,30 \\
\hline Толщина основания стремени, мм & 0,48 \\
\hline
\end{tabular}

Модуль упругости и плотность биологических тканей приведены в табл. 2.

Таблица 2

Физико-механические характеристики материалов элементов среднего уха

Table 2

Mechanical properties for components of the middle ear

\begin{tabular}{|c|c|c|}
\hline Элемент модели & Модуль упругости, МПа & 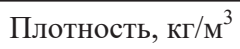 \\
\hline Область pars tensa тимпанальной мембраны & 33,4 & 1200 \\
\hline Область pars flaccida тимпанальной мембраны & 11,1 & 1200 \\
\hline Задневерхний квадрант тимпанальной мембраны & 33,4 & 1200 \\
\hline Головка молоточка & 14100 & 2550 \\
\hline Шейка молоточка & 14100 & 4530 \\
\hline Рукоятка молоточка & 14100 & 3700 \\
\hline Тело наковальни & 14100 & 2360 \\
\hline Короткий отросток наковальни & 14100 & 2260 \\
\hline Длинный отросток наковальни & 14100 & 5080 \\
\hline Стремя & 14100 & 2200 \\
\hline Тимпанальное кольцо & 0,6 & 2500 \\
\hline Наковально-молоточковое соединение & 14100 & 3200 \\
\hline Наковально-стременное соединение & 0,6 & 1200 \\
\hline Тензорное тимпанальное сухожилие & 2,6 & 2500 \\
\hline Задняя (латеральная) молоточковая связка & 0,067 & 2500 \\
\hline Передняя молоточковая связка & 21,0 & 2500 \\
\hline Верхняя молоточковая связка & 0,049 & 2500 \\
\hline Задняя связка наковальни & 0,65 & 2500 \\
\hline Передняя связка наковальни & 0,049 & 2500 \\
\hline Сухожилие стремени & 0,52 & 2500 \\
\hline Кольцевая связка стремени & 0,02 & 2500 \\
\hline
\end{tabular}

И с т очн и ки: [20-24].

Коэффициент Пуассона для всех элементов модели принимался равным 0,3. Модуль упругости хрящевого трансплантата составляет 2,8 МПа [25]. Тимпанальное кольцо упруго закреплено по боковому контуру, торцы связок зафиксированы. Также жестко заделаны узлы, расположенные на внешней поверхности кольцевой связки стремени. 
Конечно-элементное разбиение модели выполнено в полуавтоматическом режиме. Дискретная модель среднего уха в основном состоит из тетраэдрических конечных элементов типа SOLID187 (244 748 узлов и 148871 элемент). Размер ребра конечного элемента для тимпанальной мембраны составляет 0,1 мм, а для остальных элементов модели равен 0,3 мм. Отметим, что размеры конечноэлементной сетки выбраны на основании исследования сеточной сходимости модели.

Проведение операции по восстановлению целостности тимпанальной мембраны моделируется удалением задневерхнего квадранта с последующим наложением хрящевого трансплантата различной толщины. Установка трансплантата осуществляется в соответствии с протоколом, применяемым в ходе проведения хирургической операции по устранению ретракционных карманов различной стадии. Участки трансплантата, содержащие прямолинейные грани, располагаются на ножке молоточка и на неповрежденной части тимпанальной мембраны (части области pars flaccida). Участок трансплантата, содержащий криволинейную грань, располагается на тимпанальном кольце. Толщина трансплантата принимает значения 150; 250; 500 и 750 мкм. На рис. 2 приведены модели среднего уха с удаленным задневерхним квадрантом и установленным на тимпанальную мембрану хрящевым трансплантатом, имеющим толщину 750 мкм.
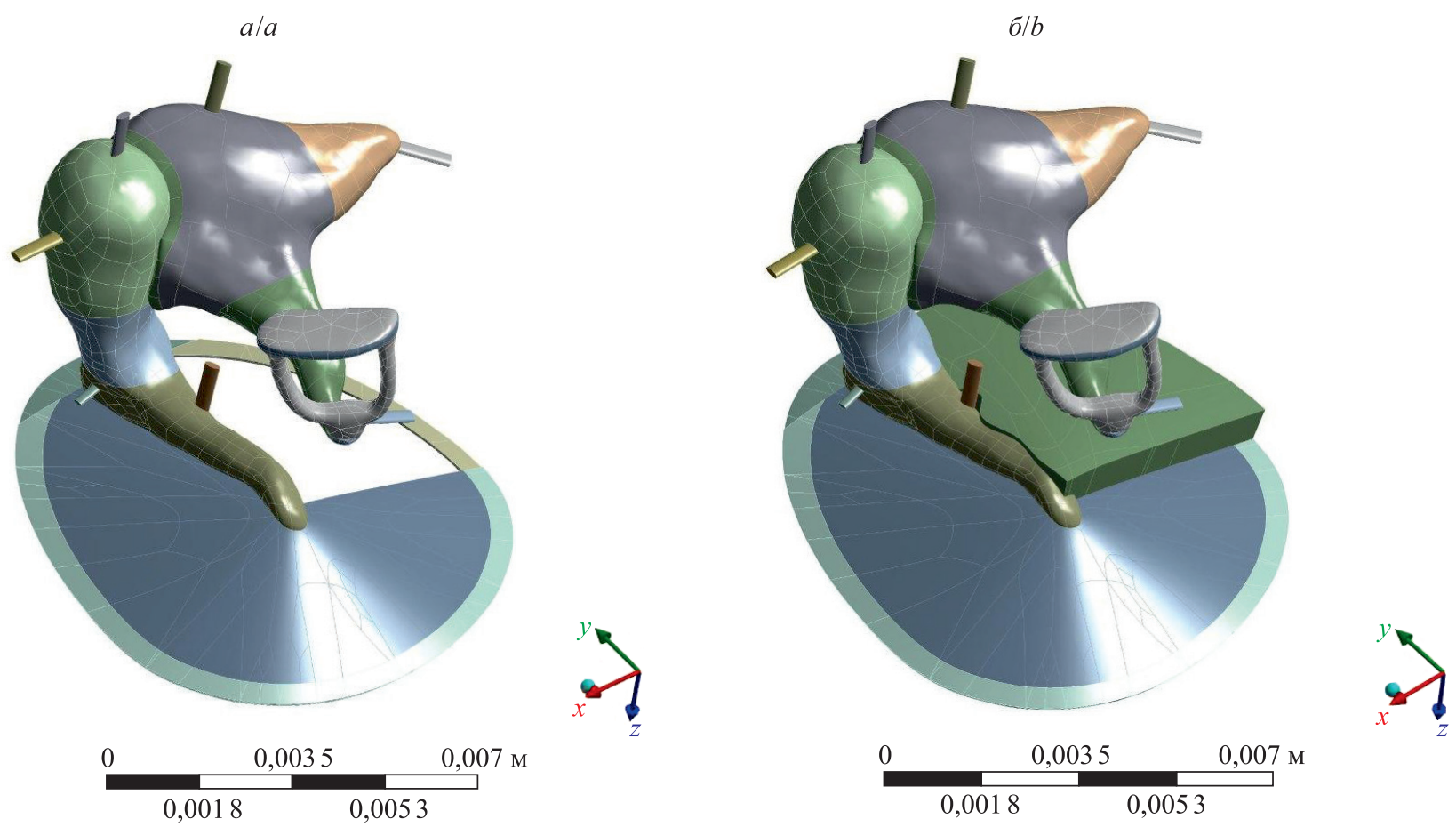

Puc. 2. Модели среднего уха с удаленным ретракционным карманом (a) и установленным хрящевым трансплантатом толщиной 750 мкм (б)

Fig. 2. Middle ear biomechanical models with removed retraction pocket (a) and attached cartilaginous graft with thickness of $750 \mu \mathrm{m}(b)$

Трансплантаты толщиной 150; 250 и 500 мкм располагаются аналогично тому, как установлен хрящевой трансплантат на рис. 2,6 .

Размер ребра конечного элемента для хрящевых трансплантатов различной толщины составляет 0,3 мм. При установке трансплантата на тимпанальное кольцо, тимпанальную мембрану и ножку молоточка использовано контактное соединение типа Bonded.

\section{Результаты и их обсуждение}

На рис. 3 представлены значения собственных частот свободных колебаний среднего уха в норме и среднего уха с установленным трансплантатом толщиной 150; 250; 500 и 750 мкм в области задневерхнего квадранта. Заметим, что при модальном анализе спектра собственных частот свободных колебаний учитываются те частоты, для которых выполняется условие $\frac{w_{\mathrm{p}}}{W_{\max }}>0,05\left(w_{\mathrm{p}}\right.$ - амплитуда колебаний центра основания стремени; $W_{\max }$ - максимальная амплитуда колебаний пупка тимпанальной мембраны). Согласно [26] собственные частоты свободных колебаний, для которых не выполняется это условие, отвечают режимам плоских колебаний или изгибным колебаниям с неподвижным стременем. Главные формы для различных мод свободных колебаний приведены на рис. 3. 


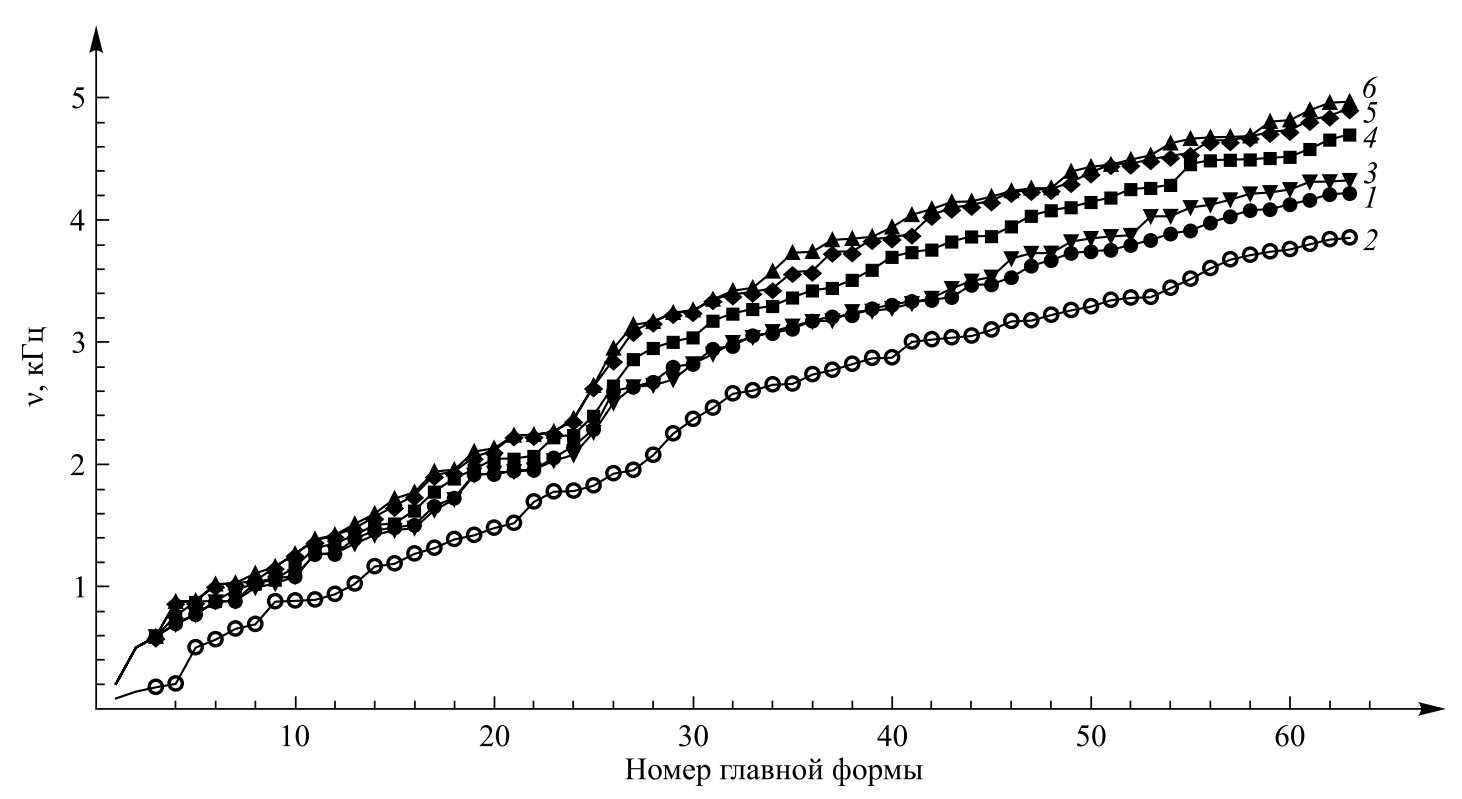

Puc. 3. Значения собственных частот свободных колебаний:

1 - среднее ухо в норме; $2,3,4,5$ и 6 - среднее ухо с установленным

хрящевым трансплантатом толщиной $100 ; 150 ; 250 ; 500$ и 750 мкм соответственно

Fig. 3. Eigenfrequency spectra: 1 - normal middle ear; 2, 3, 4,5 and 6 - middle ear with attached cartilaginous graft of $100 ; 150 ; 250 ; 500$ and $750 \mu \mathrm{m}$ thick, respectively

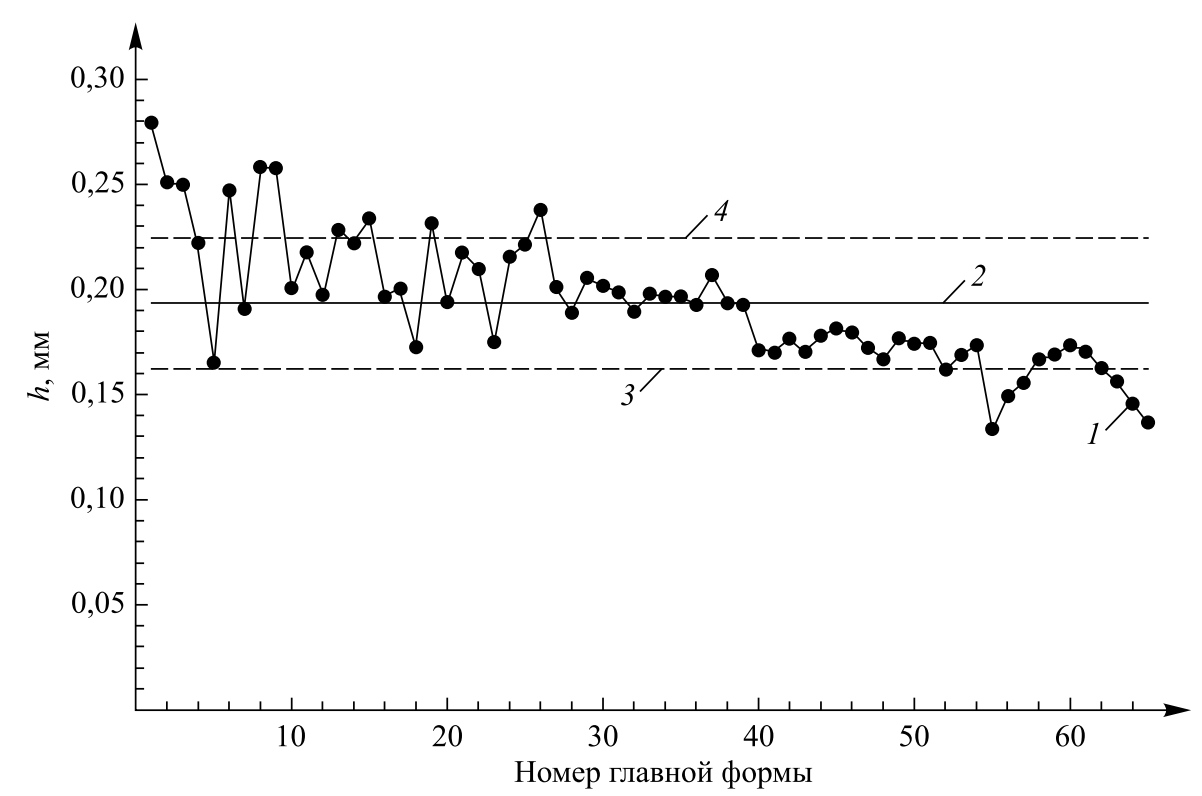

Puc. 4. Диаграмма, связывающая толщину хрящевого трансплантата с главной формой колебаний:

1 - ломаная, построенная на основании значений толщины хрящевого трансплантата, который необходимо установить на тимпанальную мембрану для приближенного

совпадения спектра собственных частот среднего уха в норме и среднего уха с трансплантатом;

2 - линия, соответствующая среднему значению толщины хрящевого трансплантата;

3,4 - линии, ограничивающие значения хрящевого трансплантата с учетом среднеквадратичного отклонения

Fig. 4. Diagram linking the cartilaginous graft thickness with the main mode of vibration:

1 - dot line plotted on the basis of thickness values of the cartilaginous graft must be attached on the tympanic membrane for approximate coincidence of the eigenfrequency spectra for normal middle ear and middle ear with the cartilaginous graft;

2 - line corresponding to the average value of the cartilage graft thickness;

3, 4 - lines limiting the values of the average cartilage graft thickness with account of standard deviation 
Из рис. 3 видно, что нижние частоты для среднего уха в норме и среднего уха с установленным хрящевым трансплантатом различной толщины практически совпадают. Это соответствует результатам работы [15], согласно которым наблюдается совпадение собственных частот свободных колебаний среднего уха с патологическими изменениями тимпанальной мембраны, а также среднего уха с патологическими изменениями в области задневерхнего квадранта и установленным хрящевым трансплантатом. Для определения толщины трансплантата используем условия равенства частот свободных колебаний для соответствующих главных форм колебаний среднего уха в норме и среднего уха с установленным на задневерхний квадрант хрящевым трансплантатом, сформулированные на основании функций регрессии. В качестве аргумента функции регрессии для каждой моды выступали толщины хрящевых трансплантатов, значениями функции являлись значения частот свободных колебаний среднего уха с хрящевым трансплантатом соответствующей толщины. Регрессия осуществлялась на основании степенной функции, среднеквадратичная погрешность при этом не превышала 4 \%.

В результате получено, что среднее значение толщины трансплантата составляет 0,193 мм, среднеквадратичное отклонение - 0,031 мм. Диаграмма, связывающая толщину хрящевого трансплантата, который следует установить на тимпанальную мембрану для приближенного совпадения спектра собственных частот среднего уха в норме и среднего уха с трансплантатом, с номером главной формы, визуализирована на рис. 4.

Из рис. 4 видно, что наиболее эффективно использование трансплантата указанной толщины сказывается на высоких частотах слухового диапазона, в частности на частотах, превышающих 2,5 кГц.

\section{Заключение}

На основании полученных результатов можно сделать вывод, что при удалении фиксированных ретракционных карманов и последующем проведении хирургической операции по восстановлению целостности тимпанальной мембраны наиболее эффективно использовать хрящевой трансплантат толщиной около 0,2 мм. Такая толщина трансплантата обеспечивает практически полное совпадение спектров собственных частот свободных колебаний среднего уха в норме и среднего уха после трансплантации. Исключение составляют низшие частоты свободных колебаний среднего уха (до 1,0 кГц), поскольку для этого диапазона частот соответствия между двумя указанными моделями среднего уха можно добиться установкой хрящевого трансплантата увеличенной толщины (около 0,25 мм). В то же время следует отметить, что использование хрящевого трансплантата толщиной 0,2 мм не приведет к существенному увеличению жесткости тимпанальной мембраны и не повлияет на изменение перемещений точек среднего уха, в частности рукоятки молоточка и основания стремени. Это подтверждается результатами экспериментальных работ [6; 24], посвященных определению частотно-амплитудной характеристики тимпанальной мембраны после установки различных трансплантатов (хрящевых пластин, хрящевых палисадов и островковых трансплантатов). Эти результаты показывают, что истонченные хрящевые пластины (в диапазоне толщин от 270 до 500 мкм) наиболее эффективны для улучшения слуховой проводимости реконструированной барабанной перепонки. Полученный в настоящей работе результат также соответствует выводам исследования [12], основанным на конечно-элементном моделировании вынужденных колебаний среднего уха с хрящевым трансплантатом различной конфигурации, установленным для устранения перфорации тимпанальной мембраны. Согласно [12] хрящевая пластина должна быть как можно более тонкой - в пределах от 0,1 до 0,2 мм. В то же время следует отметить, что результаты модального анализа моделей среднего уха в норме и среднего уха с установленным хрящевым трансплантатом толщиной 100 мкм, представленные на рис. 4 (ломаные 1, 2), показывают, что такая толщина хрящевого трансплантата не позволяет добиться совпадения спектров собственных частот свободных колебаний, причем отличия собственных частот для соответствующих главных форм достаточно существенные.

\section{Библиографические ссылки}

1. Mierzwiński J, Fishman AJ. Retraction pockets of tympanic membrane: protocol of management and results of treatment. Otorynolaryngologia. 2014;13(2):114-121.

2. Cassano M, Cassano P. Retraction pockets of pars tensa in pediatric patients: clinical evolution and treatment. International Journal of Pediatric Otorhinolaryngology. 2010;74(2):178-182. DOI: 10.1016/j.ijporl.2009.11.004.

3. Ching HH, Spinner AG, Ng M. Pediatric tympanic membrane cholesteatoma: systematic review and meta-analysis. International Journal of Pediatric Otorhinolaryngology. 2017;102:21-27. DOI: 10.1016/j.ijporl.2017.08.027.

4. Couloigner V, Molony N, Viala P, Contencin P, Narcy P, Van Den Abbeele T. Cartilage tympanoplasty for posterosuperior retraction pockets of the pars tensa in children. Otology and Neurotology. 2003;24(2):264-269. DOI: 10.1097/00129492-200303000-00022. 
5. Dornhoffer JL. Cartilage tympanoplasty. Otolaryngologic Clinics of North America. 2006;39(6):1161-1176. DOI: 10.1016/j. otc.2006.08.006.

6. Mürbe D, Zahnert T, Bornitz M, Hüttenbrink K-B. Acoustic properties of different cartilage reconstruction techniques of the tympanic membrane. Laryngoscope. 2002;112(10):1769-1776. DOI: 10.1097/00005537-200210000-00012.

7. Ермоченко СА, Михасев ГИ, Петрова ЛГ. Расчет напряженно-деформированного состояния среднего уха при его тотальной реконструкции с учетом влияния остатков тимпанальной мембраны. Российский журнал биомеханики. 2008;12(3):24-36.

8. Бегун ПИ, Грачев КВ, Ле Данг Као. Моделирование упругих свойств системы звукопроведения в норме и патологии. Сенсорные системы. 2004;18(3):206-210.

9. Бегун ПИ. Биомеханическое моделирование структур среднего уха в пакетах прикладных программ в норме, при патологических изменениях, коррекции и реконструкции. Folia Otorhinolaryngologiae et Pathologiae Respiratoriae. 2013;19(3): $43-49$.

10. Funnell WR, Khanna SM, Decraemer WF. On the degree of rigidity of the manubrium in a finite-element model of the cat eardrum. The Journal of the Acoustical Society of America. 1992;91(4):2082-2090. DOI: 10.1121/1.403694.

11. Beer H-J, Bornitz M, Hardtke HJ, Schmidt R, Hofmann G, Vogel U, et al. Modeling of components of the human middle ear and simulation of their dynamic behaviour. Audiology and Neurotology. 1999;4(3-4):156-162. DOI: 10.1159/000013835.

12. Lee C-F, Hsu L-P, Chen P-R, Chou Y-F, Chen J-H, Liu T-C. Biomechanical modeling and design optimization of cartilage myringoplasty using finite element analysis. Audiology and Neurotology. 2006;11(6):380-388. DOI: 10.1159/000095900.

13. Lee C-F, Chen J-H, Chou Y-F, Hsu L-P, Chen P-R, Liu T-C. Optimal graft thickness for different sizes of tympanic membrane perforation in cartilage myringoplasty: a finite element analysis. Laryngoscope. 2007;117(4):725-730. DOI: 10.1097/mlg.0b013e$318031 \mathrm{f0e} 7$.

14. Yu-Hsuan Wen, Lee-Ping Hsu, Peir-Rong Chen, Chia-Fone Lee. Design optimization of cartilage myringoplasty using finite element analysis. Tzu Chi Medical Journal. 2006;18(5):370-377. DOI: 10.6440/TZUCMJ.200610.0370.

15. Mikhasev G, Bosiakov S, Petrova L, Maisyuk M, Yurkevich K. Assessment of eigenfrequencies of the middle ear oscillating system: effect of the cartilage transplant. In: Awrejcewicz J, editor. Dynamical systems: modeling; 2015 December 7-10; Łódź, Poland. Cham: Springer; 2016. p. 243-254. (Springer proceedings in mathematics and statistics; volume 181). DOI: 10.1007/978-3319-42402-6 21

16. Михасев ГИ, Босяков СМ, Юркевич КС, Дутина АА, Петрова ЛГ, Майсюк ММ. Выбор толщины хрящевого трансплантата для хирургического лечения ретракционного кармана тимпанальной мембраны на основании модального анализа колебательной системы среднего уха. Журнал Белорусского государственного университета. Математика. Информатика. 2017;2:52-58.

17. Мареев ГО. Современные представления о среднем ухе и его математические модели (обзор). Саратовский научномедииинский журнал. 2012;8(1):96-100.

18. Aernouts J, Couckuyt I, Crombecq K, Dirckx JJJ. Elastic characterization of membranes with a complex shape using point indentation measurements and inverse modeling. International Journal of Engineering Science. 2010;48(6):599-611. DOI: 10.1016/j. ijengsci.2010.02.001.

19. Pengpeng Xie, Yong Peng, Junjiao Hu, Shengen Yi. A study on the effect of ligament and tendon detachment on human middle ear sound transfer using mathematic model. Proceedings of the Institution of Mechanical Engineers. Part H: Journal of Engineering in Medicine. 2019;233(8):784-792. DOI: 10.1177/0954411919853364.

20. Gan RZ, Feng B, Sun Q. Three-dimensional finite element modeling of human ear for sound transmission. Annals of Biomedical Engineering. 2004;32(6):847-859. DOI: 10.1023/B:ABME.0000030260.22737.53.

21. Sun Q, Chang K-H, Dormer KJ, Dyer RK Jr, Gan RZ. An advanced computer-aided geometric modeling and fabrication method for human middle ear. Medical Engineering and Physics. 2002;24(9):595-606. DOI: 10.1016/s1350-4533(02)00045-0.

22. Koike T, Wada H, Kobayashi T. Modeling of the human middle ear using the finite-element method. The Journal of the Acoustical Society of America. 2002;111(3):1306-1317. DOI: 10.1121/1.1451073.

23. Wever EG, Lawrence M. Physiological acoustics. Princeton: Princeton University Press; 2016. 476 p.

24. Areias B, Santos C, Jorge RMN, Gentil F, Parente MPL. Finite element modeling of sound transmission from outer to inner ear. Proceedings of the Institution of Mechanical Engineers. Part H: Journal of Engineering in Medicine. 2016;230(11):999-1007. DOI: 10.1177/0954411916666109.

25. Zahnert T, Hüttenbrink KB, Mürbe D, Bornitz M. Experimental investigation of the use of cartilage in tympanic membrane reconstruction. American Journal of Otology. 2000;21(3):322-328. DOI: 10.1016/s0196-0709(00)80039-3.

26. Mikhasev GI, Slavashevich I, Yurkevich K. Prediction of eigenfrequencies of the middle ear oscillating system after tympanoplasty and stapedotomy. In: Altenbach H, Mikhasev GI, editors. Shell and membrane theories in mechanics and biology. From macro- to nanoscale structures. Cham: Springer; 2015. p. 243-265. (Advanced structured materials; volume 45).

\section{References}

1. Mierzwiński J, Fishman AJ. Retraction pockets of tympanic membrane: protocol of management and results of treatment. Otorynolaryngologia. 2014;13(2):114-121.

2. Cassano M, Cassano P. Retraction pockets of pars tensa in pediatric patients: clinical evolution and treatment. International Journal of Pediatric Otorhinolaryngology. 2010;74(2):178-182. DOI: 10.1016/j.ijporl.2009.11.004.

3. Ching HH, Spinner AG, Ng M. Pediatric tympanic membrane cholesteatoma: systematic review and meta-analysis. International Journal of Pediatric Otorhinolaryngology. 2017;102:21-27. DOI: 10.1016/j.ijporl.2017.08.027.

4. Couloigner V, Molony N, Viala P, Contencin P, Narcy P, Van Den Abbeele T. Cartilage tympanoplasty for posterosuperior retraction pockets of the pars tensa in children. Otology and Neurotology. 2003;24(2):264-269. DOI: 10.1097/00129492-200303000-00022.

5. Dornhoffer JL. Cartilage tympanoplasty. Otolaryngologic Clinics of North America. 2006;39(6):1161-1176. DOI: 10.1016/j. otc.2006.08.006.

6. Mürbe D, Zahnert T, Bornitz M, Hüttenbrink K-B. Acoustic properties of different cartilage reconstruction techniques of the tympanic membrane. Laryngoscope. 2002;112(10):1769-1776. DOI: 10.1097/00005537-200210000-00012. 
7. Ermochenko SA, Mikhasev GI, Petrova LG. Calculation of the strain-stress state of the middle ear under its total reconstruction taking into account the influence of the tympanic membrane remnants. Rossiiskii zhurnal biomekhaniki. 2008;12(3):24-36. Russian.

8. Begun PI, Grachev KV, Le Dang Kao. Modeling springy characteristic of the sound-transfer system in rate and pathology. Sensornye sistemy. 2004;18(3):206-210. Russian.

9. Begun PI. [Biomechanical modeling of middle ear structures in applied software in normal conditions, with pathological changes, after correction and reconstruction]. Folia Otorhinolaryngologiae et Pathologiae Respiratoriae. 2013;19(3):43-49. Russian.

10. Funnell WR, Khanna SM, Decraemer WF. On the degree of rigidity of the manubrium in a finite-element model of the cat eardrum. The Journal of the Acoustical Society of America. 1992;91(4):2082-2090. DOI: 10.1121/1.403694.

11. Beer H-J, Bornitz M, Hardtke HJ, Schmidt R, Hofmann G, Vogel U, et al. Modeling of components of the human middle ear and simulation of their dynamic behaviour. Audiology and Neurotology. 1999:4(3-4):156-162. DOI: 10.1159/000013835.

12. Lee C-F, Hsu L-P, Chen P-R, Chou Y-F, Chen J-H, Liu T-C. Biomechanical modeling and design optimization of cartilage myringoplasty using finite element analysis. Audiology and Neurotology. 2006;11(6):380-388. DOI: 10.1159/000095900.

13. Lee C-F, Chen J-H, Chou Y-F, Hsu L-P, Chen P-R, Liu T-C. Optimal graft thickness for different sizes of tympanic membrane perforation in cartilage myringoplasty: a finite element analysis. Laryngoscope. 2007;117(4):725-730. DOI: 10.1097/mlg.0b013e$318031 \mathrm{f0e} 7$.

14. Yu-Hsuan Wen, Lee-Ping Hsu, Peir-Rong Chen, Chia-Fone Lee. Design optimization of cartilage myringoplasty using finite element analysis. Tzu Chi Medical Journal. 2006;18(5):370-377. DOI: 10.6440/TZUCMJ.200610.0370.

15. Mikhasev G, Bosiakov S, Petrova L, Maisyuk M, Yurkevich K. Assessment of eigenfrequencies of the middle ear oscillating system: effect of the cartilage transplant. In: Awrejcewicz J, editor. Dynamical systems: modeling; 2015 December 7-10; Lódź, Poland. Cham: Springer; 2016. p. 243-254. (Springer proceedings in mathematics and statistics; volume 181). DOI: 10.1007/978-3319-42402-6 21.

16. Mikhasev GI, Bosiakov SM, Yurkevich KS, Dutina AA, Petrova LG, Maisyuk MM. Graft thickness assessment for surgery of retraction pocket of the middle ear based on finite-element analysis of eigenfrequencies of the eardrum oscillating system. Journal of the Belarusian State University. Mathematics and Informatics. 2017;2:52-58. Russian.

17. Mareev GO. Modern mathematical models of middle ear (review). Saratov Journal of Medical Scientific Research. 2012; 8(1):96-100. Russian.

18. Aernouts J, Couckuyt I, Crombecq K, Dirckx JJJ. Elastic characterization of membranes with a complex shape using point indentation measurements and inverse modeling. International Journal of Engineering Science. 2010;48(6):599-611. DOI: 10.1016/j. ijengsci.2010.02.001.

19. Pengpeng Xie, Yong Peng, Junjiao Hu, Shengen Yi. A study on the effect of ligament and tendon detachment on human middle ear sound transfer using mathematic model. Proceedings of the Institution of Mechanical Engineers. Part H: Journal of Engineering in Medicine. 2019;233(8):784-792. DOI: 10.1177/0954411919853364.

20. Gan RZ, Feng B, Sun Q. Three-dimensional finite element modeling of human ear for sound transmission. Annals of Biomedical Engineering. 2004;32(6):847-859. DOI: 10.1023/B:ABME.0000030260.22737.53.

21. Sun Q, Chang K-H, Dormer KJ, Dyer RK Jr, Gan RZ. An advanced computer-aided geometric modeling and fabrication method for human middle ear. Medical Engineering and Physics. 2002;24(9):595-606. DOI: 10.1016/s1350-4533(02)00045-0.

22. Koike T, Wada H, Kobayashi T. Modeling of the human middle ear using the finite-element method. The Journal of the Acoustical Society of America. 2002;111(3):1306-1317. DOI: 10.1121/1.1451073.

23. Wever EG, Lawrence M. Physiological acoustics. Princeton: Princeton University Press; 2016. 476 p.

24. Areias B, Santos C, Jorge RMN, Gentil F, Parente MPL. Finite element modeling of sound transmission from outer to inner ear. Proceedings of the Institution of Mechanical Engineers. Part H: Journal of Engineering in Medicine. 2016;230(11):999-1007. DOI: 10.1177/0954411916666109.

25. Zahnert T, Hüttenbrink KB, Mürbe D, Bornitz M. Experimental investigation of the use of cartilage in tympanic membrane reconstruction. American Journal of Otology. 2000;21(3):322-328. DOI: 10.1016/s0196-0709(00)80039-3.

26. Mikhasev GI, Slavashevich I, Yurkevich K. Prediction of eigenfrequencies of the middle ear oscillating system after tympanoplasty and stapedotomy. In: Altenbach H, Mikhasev GI, editors. Shell and membrane theories in mechanics and biology. From macro- to nanoscale structures. Cham: Springer; 2015. p. 243-265. (Advanced structured materials; volume 45). 